\title{
A structural encoding technique for the synthesis of asynchronous circuits*
}

\author{
Josep Carmona $^{1} \quad$ Jordi Cortadella $^{1} \quad$ Enric Pastor $^{2}$ \\ ${ }^{1}$ Software Department \\ Universitat Politècnica de Catalunya \\ Barcelona, Spain \\ $\{$ jcarmona, jordic $\} @ 1$ si.upc.es \\ ${ }^{2}$ Computer Architecture Department \\ Universitat Politècnica de Catalunya \\ Barcelona, Spain \\ enric@ac.upc.es
}

\begin{abstract}
This paper presents a method for the automatic synthesis of asynchronous circuits from Petri net specifications. The method is based on a structural encoding of the system in such a way that a circuit implementation is always guaranteed. Moreover, a set of transformations is presented for the subclass of Free-Choice Petri nets that enables the exploration of different solutions. All transformations preserve the property of free-choiceness, thus enabling the use of structural methods for the synthesis of asynchronous circuits. Preliminary experimental results indicate that the quality of the circuits is comparable to that obtained by methods that require an exhaustive enumeration of the state space.

This novel synthesis method opens the door to the synthesis of large control specifications generated from hardware description languages.
\end{abstract}

\section{Introduction}

In the last few years, there has been an increasing interest in asynchronous circuits. Potential advantages, such as modularity, absence of clock skew problems, average performance and low power, have encouraged many researchers and designers to devote some efforts in understanding and proposing techniques for asynchronous circuit design [9].

If some unanimity exists about asynchronous circuits, it is that they are difficult to design. The absence of clock does not allow a discrete abstraction of time and, therefore, the behavior of any signal at any instant can be relevant for the

* This work has been partially funded by CICYT (TIC 98-0410 and TIC 98-0949), ACiD-WG (ESPRIT 21949), a grant by Intel Corporation, and CIRIT (1999SGR-150 and 2000FI-00472). correctness of the circuit. A significant effort has been spent in studying and proposing automatic synthesis techniques that can alleviate the burden of designing asynchronous circuits. This paper focuses on techniques for the synthesis of control circuits.

Currently, there are several academic tools that work at the logic level and attempt to optimize the resulting circuit by using variations of the state-of-the-art Boolean minimization techniques [12, 6, 22]. Given that asynchronous circuits are typically modeled as concurrent systems, the existing synthesis approaches often suffer from the state explosion problem derived from concurrency.

A crucial problem of most asynchronous logic synthesis tools is that they are not always capable of deriving an implementation from the specification. The main reason for that is that some of the implementation properties must be ensured by transforming the specification. And this task is performed automatically by using heuristics that cannot explore the complete space of configurations.

Direct translation methods that do not exploit the power of Boolean minimization have also been proposed [11, 4, 1 , 17]. This type of strategies guarantees an implementation by construction, but does not exploit the potential optimizations that can be performed at logic level. Typically, the size of the obtained circuits is linear on the size of the specification.

There have been few attempts to combine both approaches [25, 15]. However, direct translation methods usually generate circuit structures that cannot be locally transformed to derive succinct representations of the same behavior. For this reason, the results obtained by these methods are comparable to peephole optimizations realized on the original structures.

Nowadays, the knowledge of asynchronous techiques have reached a level of maturity that have enabled some researchers to face the problem of synthesis from Hardware 
Description Languages (HDLs), such as Verilog [3, 19] or VHDL [27]. This new trend also implies dealing with control circuits that are both large and well-structured.

Due to the aforementioned state explosion problem, there exist severe limitations on the size of the specifications that can be handled by existing synthesis tools. However, the fact that control specifications derived from HDLs tend to be well-structured opens the door to use techniques that do not require an explicit representation of the state space.

This paper presents some contributions into that direction, with the aim that automatic synthesis techniques based on the presented theoretical results will be proposed in the future. The concurrent model used in this paper is based on Petri nets [21]. The main contribution consists in proposing a set of structural transformations of the specification that guarantees an implementation of the behavior without explicitly enumerating the state space of the system. The transformations are proposed for the subclass of Free-choice Petri nets. This subclass seems to be a good trade-off between the expresiveness power required by well-structured control specifications and the methods that can manipulate them without suffering from the size of the state space.

Moreover, the presented transformations preserve the structural properties of the specification, thus enabling the use of logic synthesis techniques that do not require an explicit representation of the state space [24].

The paper is organized as follows. Section 2 describes previous and related work. Section 3 presents basic definitions and background used along the paper. The encoding method and its properties is presented in Section 4. The property-preserving transformations are described in Section 5. Finally, Section 6 illustrates the method with an example and reports some preliminary results.

\section{Related work and overview}

Signal Transition Graphs (STGs) [26, 5] are interpreted Petri nets used for the specification and synthesis of asynchronous controllers. In STGs, transitions represent rising and falling signal transitions, denoted by positive and negative events (e.g. $a+, a-$ ). Several techniques that circumvent the state explosion problem have been proposed for the synthesis from STGs. However, most of them only work for marked graphs, a very restrictive class of specifications that cannot model choice behaviors [13].

To the best of our knowledge, the only work in this area that has covered the synthesis of specifications with Freechoice Petri nets was presented in [24]. Besides allowing the specification of choice, Free-choice Petri nets also have nice structural properties that enable the use of polynomial algorithms to analyze their behavior [10].

Unfortunately, none of the methods mentioned before has been able to effectively tackle the problem of finding an encoding of the specification that guarantees an implementation. Even the known structural methods working for some subclasses of STGs rely on the fact that heuristics with affordable computational cost will find a solution with high probability [29, 23].

The encoding problem is illustrated in Figure 1. Given a specification (Figure 1(a)), each state of the reachability graph is assigned a binary vector that represents the value of each signal at that state (Figure 1(b)). For a circuit to be derived from the specification, it is required that the value of the signals can uniquely distinguish non-equivalent states. In this example, there are two states that cannot be distinguished by their codes (shadowed in the figure). Solving the state encoding problem is usually performed by adding new signals in the specification that preserve implementation properties. Doing so is not an easy task [7].

The method presented in this paper has been inspired on previous work for the direct synthesis of circuits from Petri nets. One of the relevant techniques was proposed in [28], where a set of cells that mimic the token flow in Petri nets was designed. The circuit was built by abutting the cells and producing a structure isomorphic to the Petri net. This type of cells, called David cells, were initially proposed in [8].

Figure 2 depicts a very simple example on how these cells can be abutted to build a distributor that controls the propagation of activities along a ring. The behavior of one of the cells in the distributor can be summarized by the following sequence of events:

$$
\begin{gathered}
\cdots \rightarrow \underbrace{c_{i-1}-}_{\begin{array}{c}
i \text {-th cell } \\
\text { excitation }
\end{array}} \rightarrow \underbrace{a_{i}+\rightarrow \hat{a}_{i}-}_{i \text {-th cell setting }} \rightarrow \\
\rightarrow \underbrace{\hat{a}_{i-1}+\rightarrow a_{i-1}-\rightarrow c_{i-1}+}_{(i-1) \text {-th cell resetting }} \rightarrow \underbrace{c_{i}-}_{\begin{array}{c}
(i+1) \text {-th cell } \\
\text { excitation }
\end{array}} \rightarrow \cdots
\end{gathered}
$$

In [28], each cell was used to represent the behavior of one of the transitions of the Petri net. The approach presented in this paper is based on encoding the system by inserting a new signal for each place with a behavior similar to a David cell. With such an encoding approach, two goals are achieved:

- A solution for the encoding problem is guaranteed at the expense of over-encoding the states of the system.

- The structural properties of the specification are preserved, thus enabling the use of transformations to optimize the resulting circuit.

In the forthcoming sections, the encoding method and a set of optimizing transformations are discussed. 


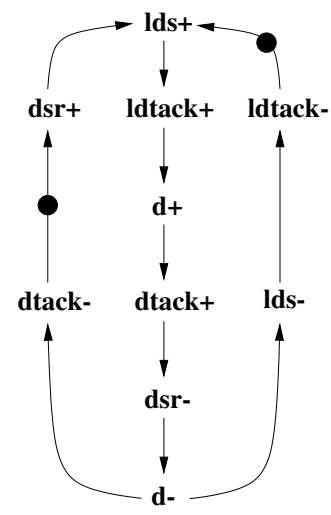

(a)

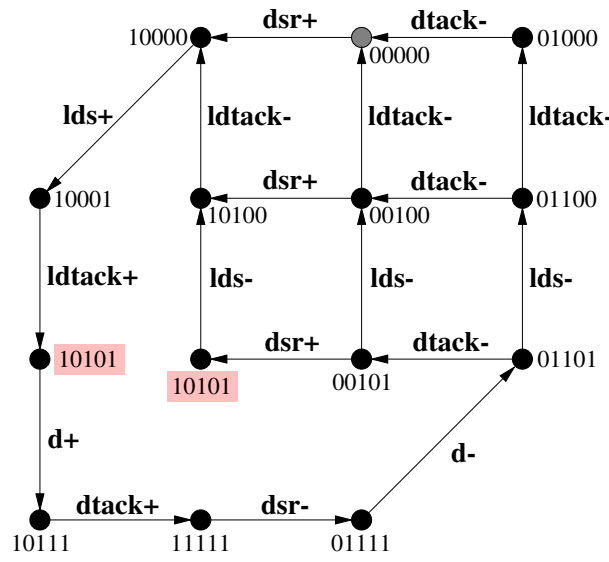

(b)

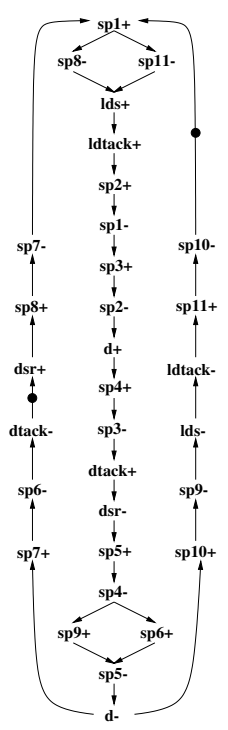

(c)

Figure 1. (a) STG, (b) Encoded graph (<dsr, dtack, ldtack, d, lds $>$ ), (c) Structurally encoded STG.

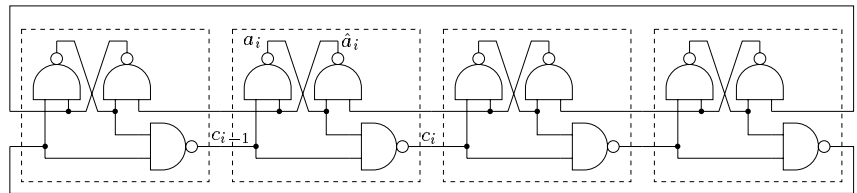

Figure 2. Distributor built from David cells [14].

\section{Petri Nets and Signal Transition Graphs}

The theory presented in this paper holds for the class of consistent and deterministic Signal Transition Graphs with an underlying Free-choice live and safe Petri net and initial home marking. The necessary definitions to support the theory are next presented.

\subsection{Petri Nets}

A Petri Net (PN) is a 4-tuple $\Sigma=\left\langle\mathcal{P}, \mathcal{T}, \mathcal{F}, M_{0}\right\rangle$, where $\mathcal{P}$ is the set of places, $\mathcal{T}$ is the set of transitions, $\mathcal{F} \subseteq(\mathcal{P} \times$ $\mathcal{T}) \cup(\mathcal{T} \times \mathcal{P})$ is the flow relation, and $M_{0}$ is the initial marking. A marking of a $\mathrm{PN}$ is an assignment of a nonnegative integer to each place. If $k$ is assigned to place $p$ by marking $M$ (denoted $M(p)=k$ ), we will say that $p$ is marked with $k$ tokens. Given a node $x \in \mathcal{P} \cup \mathcal{T}$, its post-set and pre-set are denoted by ${ }^{\bullet} x$ and $x^{\bullet}$ respectively.

A path in a PN is a sequence $u_{1} \ldots u_{r}$ of nodes such that $\forall i, 1 \leq i<r:\left(u_{i}, u_{i+1}\right) \in \mathcal{F}$. A path is called simple if no node appears more than once on it.

A transition $t$ is enabled in a marking $M$ when all places in $\bullet$ are marked. When a transition $t$ is enabled, it can fire by removing a token from each place in ${ }^{\bullet} t$ and putting a token to each place in $t^{\bullet}$. A marking $M^{\prime}$ is reachable from $M$ if there is a sequence of firings $t_{1} t_{2} \ldots t_{n}$ that transforms $M$ into $M^{\prime}$, denoted by $M\left[t_{1} t_{2} \ldots t_{n}\right\rangle M^{\prime}$. A sequence of transitions $t_{1} t_{2} \ldots t_{n}$ is a feasible sequence if it is firable from $M_{0}$. The set of reachable markings from $M_{0}$ is denoted by $\left[M_{0}\right\rangle$. A marking is a home marking if it is reachable from every marking of $\left[M_{0}\right\rangle$.

A place in a PN is redundant if its elimination does not change the behavior of the net. A PN is place-irredundant if it does not have redundant places.

A PN is live iff every transition can be infinitely enabled through some feasible sequence of firings from any marking in $\left[M_{0}\right\rangle$. A PN is safe if no marking in $\left[M_{0}\right\rangle$ assigns more than one token to any place. A Free-Choice Petri net is a PN such that if $(p, t) \in \mathcal{F}$ then $\bullet \times p^{\bullet} \subseteq \mathcal{F}$, for every place $p$ [10]. In the rest of the paper, we will deal with Free-choice live and safe Petri nets (FCLSPN).

Checking for liveness, safeness and redundant places can be done in polynomial time for Free-choice Petri nets [10].

\subsection{Signal Transition Graphs}

A Signal Transition Graph (STG) [26] is a triple $\langle\Sigma, A, \Lambda\rangle$, where $\Sigma$ is a PN, $A$ is a set of signals, partitioned into input signals $\left(A_{I}\right)$, output signals $\left(A_{O}\right)$, and internal signals $\left(A_{I N T}\right)$, and $\Lambda$ is the labeling function 
$\Lambda: \mathcal{T} \rightarrow(A \times\{+,-\}) \cup\{\varepsilon\}$, where all transitions not labeled with the silent event $(\varepsilon)$ are interpreted as signal changes. Rising and falling transitions of a signal $a \in A$ are denoted by $a+$ and $a-$, respectively, while $a *$ denotes a generic rising or falling transition ${ }^{1}$.

An example of STG is shown in Figure 1(a). For simplicity, those places that only have one predecessor and one successor transition are not depicted. In that case, the tokens are held on the corresponding arcs.

\subsection{Observational equivalence}

The notion of observational equivalence, as defined by Milner [18], with respect to a set of observable events is relevant in this paper. Informally, two systems are observationally equivalent if their behavior cannot be distinguished by interacting with them. When necessary, we will consider observational equivalence with respect to input and output signals (not internal), or with respect to all signals. The following definitions assume observational equivalence with respect to all signals.

An STG is deterministic if the firing of two different transitions with the same label in a marking $M \in\left[M_{0}\right\rangle$ leads to observational equivalent markings.

A signal is said to be enabled in a marking $M$ if there is a marking $M^{\prime}$ which is observationally equivalent to $M$ and a transition of the signal is enabled in $M^{\prime}$ (as a particular case, $M=M^{\prime}$ ).

\subsection{Concurrency and ordering relations}

A pair of transitions $t_{i}, t_{j} \in \mathcal{T}$ are said to be concurrent if there is a marking $M \in\left[M_{0}\right\rangle$ such that $M\left[t_{j} t_{i}\right\rangle$ and $M\left[t_{i} t_{j}\right\rangle$. The concept of concurrency can be extended to signals. Two signals $a$ and $b$ are said to be concurrent if there are two transitions with labels $a *$ and $b *$ that are concurrent.

An STG is non-autoconcurrent if it does not contain any pair of concurrent transitions of the same signal. An STG satisfies the consistency condition if it is nonautoconcurrent and the signal changes in every feasible sequence of signal transitions alternate. This last condition restricts the feasible sequences: the change $0 \rightarrow 1(1 \rightarrow 0)$ can only be followed by the change $1 \rightarrow 0(0 \rightarrow 1)$ for each signal appearing in a feasible sequence.

\subsection{Encoding}

Each marking of an STG is encoded with a binary vector of signal values by means of a labeling function $\lambda:\left[M_{0}\right\rangle \rightarrow$ $\{0,1\}^{|A|}$. All markings must be consistently encoded by

\footnotetext{
${ }^{1}$ Along this paper, we will often use the label of a transition to denote the transition itself.
}

$\lambda$, i.e. no marking $M$ can have an enabled rising (falling) transition $a+(a-)$ if $\lambda(M)_{a}=1\left(\lambda(M)_{a}=0\right)$.

Figure 1(b) depicts the set of reachable states derived from the STG in Figure 1(a), with the corresponding encoding.

An STG is said to satisfy the complete state coding (CSC) property if, when the same binary code is assigned to two different markings, the set of internal and output signals enabled at each marking is the same. The STG in Figure 1(a) does not satisfy the CSC property, since there are two different markings with the code 10101, and two output transitions, $d+$ and $l d s-$, only enabled in one of them.

A more restrictive property, called unique state coding (USC), holds if all reachable markings are assigned a unique binary code, i.e., $\forall M_{1}, M_{2} \in\left[M_{0}\right\rangle: M_{1} \not M_{2} \Rightarrow$ $\lambda\left(M_{1}\right) \neq \lambda\left(M_{2}\right)$, where $\sim$ denotes observational equivalence.

The CSC property is a necessary condition for the correct implementation of an STG specification. When the CSC condition holds, the events that the circuit must produce at each reachable state are uniquely determined by the binary code of the state itself.

\subsection{Synthesis of speed-independent circuits}

Here, we briefly sketch how a circuit can be derived from an STG. This theory is valid for the class of speedindependent circuits, which are correct when assuming that all components of the circuit can have any delay [20].

If we call $a_{1}, \ldots, a_{n}$ the signals of the circuit, each noninput signal $x$ can be implemented by a gate that realizes a logic function $f_{x}$. The logic function is defined for each binary vector $v \in\{0,1\}^{n}$ as follows:

$f_{x}(v)=\left\{\begin{array}{cc}1 & \text { if } \exists M: \lambda(M)=v \wedge(\text { some } x+\text { enabled in } M \vee \\ & \left.\left(\lambda(M)_{x}=1 \wedge \text { no } x-\text { enabled in } M\right)\right) \\ 0 \quad \text { if } \exists M: \lambda(M)^{2}=v \wedge(\text { some } x-\text { enabled in } M & \\ & \left.\left(\lambda(M)_{x}=0 \wedge \text { no } x+\text { enabled in } M\right)\right) \\ -\quad \text { if } \nexists M: \lambda(M)=v\end{array}\right.$

In case the CSC property does not hold, the previous definition is ambiguous, since a binary vector could be found for which there are two different markings that would make $f_{x}$ equal to 0 and 1 simultaneously.

The previous function is incompletely specified. For those vectors in which $f_{x}(v)=-$, the function may take any value, since those vectors will never appear in any reachable state of the system. This set of vectors define the don't care set of the function, which is extremely important for an efficient Boolean minimization.

\section{Structural Encoding}

This section presents a transformation applied to STGs. The features of this transformation are the following: 
- It guarantees the USC property.

- It preserves free-choiceness.

- It preserves consistency, liveness, safeness, initial home marking and observational equivalence with respect to the input and output signals.

- It has linear complexity on the size of the STG.

This is the first method that guarantees a solution for the encoding problem and tackles the problem in linear complexity for the class of FCLSPNs. The transformation is based on the insertion of a signal for each place of the STG that mimics the token flow on that place.

Althought the encoding transformation does not require the net to be free choice, it preserves this property. This is important in our framework to enable the use of structural methods for synthesis.

The transformations will be presented as a rule to be applied to the transitions of the STG. Before the application of the Structural Encoding, the set of signals of the STG has been augmented with one signal $s p$ for each place $p$ of the STG. In order to simplify the presentation of the rules and the corresponding proofs, we will use silent transitions on the definition of the rules.

\subsection{Encoding transformation}

Let $S=\left\langle\left\langle\mathcal{P}, \mathcal{T}, \mathcal{F}, M_{0}\right\rangle, A, \Lambda\right\rangle$ be an STG with underlying FCLSPN and initial home marking. The Structural Encoding of $S$ derives the STG Enc $(S)$ in which a new internal signal $s p$ has been created for each place $p \in \mathcal{P}$, and the transformation rule described in Figure 3 has been applied to each transition $t \in \mathcal{T}$. The new transtions appearing in $\operatorname{Enc}(S)$, labelled with $s p *$, will be called E-transitions along the paper.

Let us now prove properties on $\operatorname{Enc}(S)$.

Proposition 4.1 Enc $(S)$ is free-choice.

Proof: Every new place $p$ appearing in $\operatorname{Enc}(S)$ has $|\bullet p|=\left|p^{\bullet}\right|=1$ by construction. The original places in $\bullet$ and $t^{\bullet}$ keep the same flow relations with the rest of the net. Therefore, the underlying PN of $E n c(S)$ is free-choice.

Proposition 4.2 $\operatorname{Enc}(S)$ is live, safe, has initial home marking and is observational equivalent to $S$ with respect to the input and output signals.

Proof: The transformation for structural encoding is a trivial combination of a set of transformations proposed by Berthelot that preserve liveness, safeness and home marking [2]. These transformations also preserve the behavior condition, i.e. each conflict resolution in $\operatorname{Enc}(S)$ is performed by some observable transition.
1. Create the silent transitions $\varepsilon_{1}$ and $\varepsilon_{2}$.

2. For each place $p \in{ }^{\bullet} t$, create a new transition with label $s p-$ and insert new arcs and places for creating a simple path from $\varepsilon_{1}$ to $\varepsilon_{2}$, passing through $s p-$.

3. For each place $p \in t^{\bullet}$, substitute the $\operatorname{arc}(t, p)$ by the arc $\left(\varepsilon_{2}, p\right)$, create a new transition labeled as $s p+$ and insert new arcs and places for creating a simple path from $t$ to $\varepsilon_{1}$, passing through $s p+$

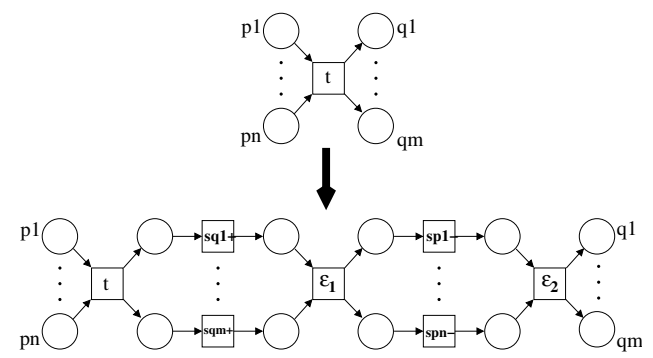

Figure 3. Transformation rule for each transition $t \in \mathcal{T}$.

From the behavior condition, it immediately follows that observational equivalence is also preserved.

Proposition $4.3 \mathrm{Enc}(S)$ is consistent.

Proof: Given that the observational equivalence is preserved, consistency directly holds for the signals already in $S$. It only remains to prove that it also holds for the Etransitions of the new inserted signals.

By construction, the new $s p$ signals mimic the token flow in places. Given that the dynamic behavior corresponds to a safe PN, no more than two consecutive rising or falling transitions can occur for these signals.

Proposition 4.4 Enc(S) has the USC property.

Proof: We will prove that each marking of $\operatorname{Enc}(S)$ is uniquely identified by a binary vector of all signals. Figure 4 depicts a fragment of $\operatorname{Enc}(S)$ that results from applying the transformation rule to a transition with $p_{1} \ldots p_{n}$, and $q_{1} \ldots q_{m}$ as predecessor and successor places, respectively. Without loss of generality, we will assume that the label of the transition is $x+$, and that place $q_{i}$ has one successor transition with label $y+$. With two exceptions that will be discussed later, the marking of all places in the picture can be uniquely determined as follows:

$$
\begin{aligned}
& M\left(a_{i}\right)=1 \quad \Leftrightarrow \quad s q_{i}=0 \wedge s p_{1}=\cdots=s p_{n}=1 \wedge x=1 \\
& M\left(b_{i}\right)=1 \quad \Leftrightarrow \quad s q_{i}=1 \wedge s p_{1}=\cdots=s p_{n}=1 \wedge x=1 \\
& M\left(c_{i}\right)=1 \quad \Leftrightarrow \quad s p_{i}=1 \wedge s q_{1}=\cdots=s q_{m}=1 \wedge x=1 \\
& M\left(d_{i}\right)=1 \quad \Leftrightarrow \quad s p_{i}=0 \wedge s q_{1}=\cdots=s q_{m}=1 \wedge x=1 \\
& M\left(q_{i}\right)=1 \quad \Leftrightarrow \quad s q_{i}=1 \wedge y=0 \wedge x=1
\end{aligned}
$$

When defining the previous equations, it is important to use the fact that the STG is safe, consistent and that transitions of the same signal cannot be concurrent. We will only 


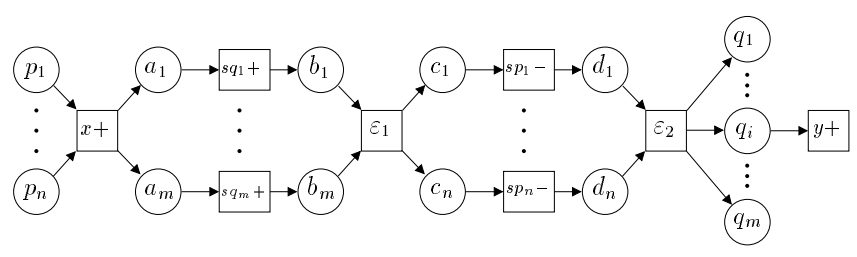

Figure 4. Place encoding to guarantee USC.

prove the equality for $M\left(a_{i}\right)$. The other equalities can be proved in a similar way.

$\Rightarrow$

$M\left(a_{i}\right)=1$ implies $s q_{i}=0$, since $s q_{i}+$ is enabled. Otherwise the STG would not be consistent. $M\left(a_{i}\right)=1$ also implies $s p_{1}=\cdots=s p_{n}=1$, since the liveness and safeness of the STG imply that $\varepsilon_{1}$ has not fired after $x+$ has fired. Therefore, none of the $s p_{i}-$ transitions has fired yet, while all $s p_{i}+$ transitions already fired before $x+$. Finally, $M\left(a_{i}\right)=1$ clearly implies $x=1$.

$\Leftarrow$

By the consistency of signal $x$, the only markings in which $s p_{1}=\cdots=s p_{n}=1$ and $x=1$ correspond to markings in which some tokens are held in the places after $x+$ but before $s p_{1}-\ldots s p_{n}-$. The fact that $s q_{i}=0$ implies that place $a_{i}$ has a token.

As mentioned before, there are two exceptions in which the binary code does not uniquely identify the marking. One exception corresponds to the submarkings in which $M\left(b_{1}\right)=\cdots=M\left(b_{m}\right)=1$ and $M\left(c_{1}\right)=\cdots=$ $M\left(c_{n}\right)=1$, respectively. These submarkings are only separated by a silent transition, $\varepsilon_{1}$, that makes them observationally equivalent. Therefore, the USC property is still preserved. The other exception corresponds to the submarkings separated by $\varepsilon_{2}$.

\subsection{Preserving the Input/Output Interface}

Preserving the observational equivalence with respect to the input and output signals of the specification is not sufficient to guarantee a correct implementation of a system. When one wants to implement a module of a system as a circuit, the input/output interface for that module is typically fixed a priori. From the point of view of the circuit, the environment can be considered as another module with mirrored signals (input and outputs of the circuit are outputs and inputs of the environment).

Since the environment must be considered as an already implemented system that cannot change its interface, the causality relations between the outputs of the circuit and the inputs of the environment must be preserved. In practice, this means that if the firing of an output signal may enable an input signal, then this causality must be preserved along any transformation of the specification.
The previous condition has been formalized under the notion of I/O equivalence [16]. This paper will not describe the details of this equivalence. However, a set of constraints on the STGs are imposed to allow the use of another transformation that preserves I/O equivalence.

In particular, an io-STG is defined as an STG with the following constraints: (a) any choice place (with more than one successor) must only precede transitions of input signals; (b) the firing of one input signal cannot immediately enable the firing of another input signal.

First condition indicates that choices are only decided by the environment. Second condition imposes the fact that the predecessors of input transitions are always output transitions. With these constraints, the transformation rule shown in Figure 5 can be applied to any transition of a non-input signal. For input transitions, the previous transformation presented in Figure 3 is applied.

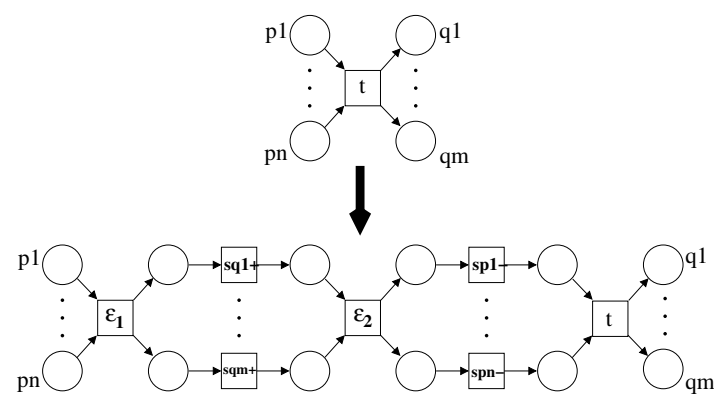

Figure 5. Transformation rule for non-input signals to preserve the $1 / 0$ interface.

Note that the two transformations only differ on the location of the E-transitions. For non-input signals, the Etransitions precede the transformed transition. In this way, the creation of new causality relations from E-transitions to input transitions is avoided and, thus I/O equivalence preserved.

The proofs for preserving free-choiceness, liveness, safeness and observational equivalence are similar to those presented in the previous section when applied to the class of io-STGs.

Figure 6 depicts an example of the structural encoding by applying the transformations presented in this section.

\section{Design space exploration}

Even though the encoding method previously presented guarantees an implementation of the system, the insertion of an internal signal for each place may be too costly, in size and performance, for the final circuit.

This section presents a kit of structural transformations that aim at the exploration of the design space. Behind these transformations, we assume to have a synthesis framework 
that can evaluate the cost of the implementation in polynomial complexity on the size of the specification [24]. This framework is also capable of detecting CSC conflicts with low complexity [23]. The transformations are mainly meant to deal with the new inserted E-transitions.

The kit of transformations we present are not new in the literature. They have been proposed by other authors (see $[2,21,10]$ ) or can be obtained by combining several of those transformations. The ones that have been used in this work are next described. All of them preserve the relevant properties of the STGs required for this work.
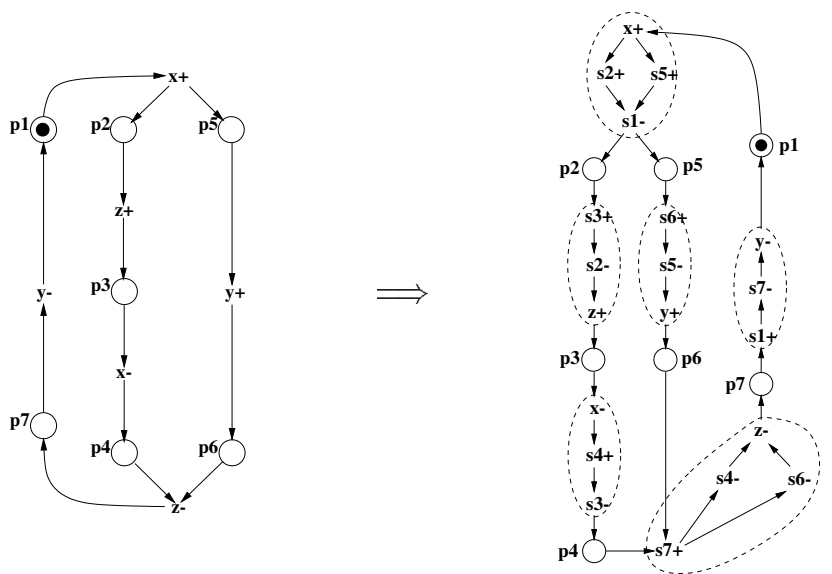

Figure 6. Structural encoding ( $x$ is input and $y$ and $z$ are outputs).

Concurrency reduction. Given two concurrent transitions, $t_{i}$ and $t_{j}$, such that $\bullet\left(\bullet_{i}\right) \cap \bullet\left(\bullet t_{j}\right) \neq \emptyset$, concurrrency is reduced by including two places that force an alternation on the firing of the two transitions (see Figure 7(a)).

Increase of concurrency. Given two ordered transitions, $t_{i}$ and $t_{j}$, such that $t_{i}^{\bullet}={ }^{\bullet} t_{j}=\{p\}$, two parallel branches are created so that they can be fired concurrently. This transformation can be obtained by combining some of the ones presented in [21] (initially proposed in [2]). The transformation is shown in Figure 7(b).

Elimination of signal. Given one of the internal signals of the STG, it can be eliminated by changing the label of all transitions of that signal and making them silent $(\varepsilon)$. This transformation is only accepted when the removal of the signal does not create CSC conflicts in the specification.

Elimination of silent transitions. Some of the transformations may insert silent transitions in the specification. By removing them, the size of specification can be reduced and the synthesis algorithms sped-up. However, the elimination the transition requires the substitution of $n+m$ places (predecessor and successor) by $n \cdot m$ places (see Figure 7(c)). Heuristics can be used to determine when the elimination can be useful.

Elimination of redundant places. Some of the places may be eliminated without changing the behavior of the net. Linear programming techniques can be used to determine when places are redundant $[2,10]$.

On top of this kit of transformations, a search engine is expected to explore the design space. Greedy heuristics or optimization techniques such as simulated annealing, genetic algorithms or tabu search can be used to explore different configurations. All these techniques require a fast estimation of the cost of the explored configurations. The cost function can be efficiently supported by the polynomial algorithms that can be typically used to manipulate free-choice Petri nets.

\section{Example and experimental results}

The transformations presented in this paper have been applied to well-known specifications from the literature of asynchronous circuit design.

Currently, no search engine is still available to apply the transformations automatically. Instead, they are applied $m e$ chanically with the intervention of the designer that, at each step, chooses a transformation that intuitively leads to a better implementation.

In more detail, the synthesis strategy consists of the following steps:

1. Apply the encoding transformations on all transitions, as explained in Section 4.

2. Iteratively and greedily apply the transformation elimination of signal to all internal signals as far as no CSC conflicts appear. In general, some of the internal signals will remain in the specification.

3. Iteratively apply transformations and evaluate the implementation cost of the new specification. Accept any transformation that produces a cost improvement. The cost is evaluated as the number of literals of the Boolean equations implementing the circuit.

4. Stop when no transformation can be applied to improve the cost of the circuit.

Given that the previous method has not been automated yet, the application of the transformations at each step has not been done in an exhaustive manner. Structural methods are used for checking CSC and deriving boolean equations [24].

The results have been compared with those obtained by the tool petrify [6], that does an explicit enumeration of the state space, thus suffering from the state explosion problem.

\subsection{A case study: adfast}

This example corresponds to specification of an analogic-to-digital fast converter with three input signals 

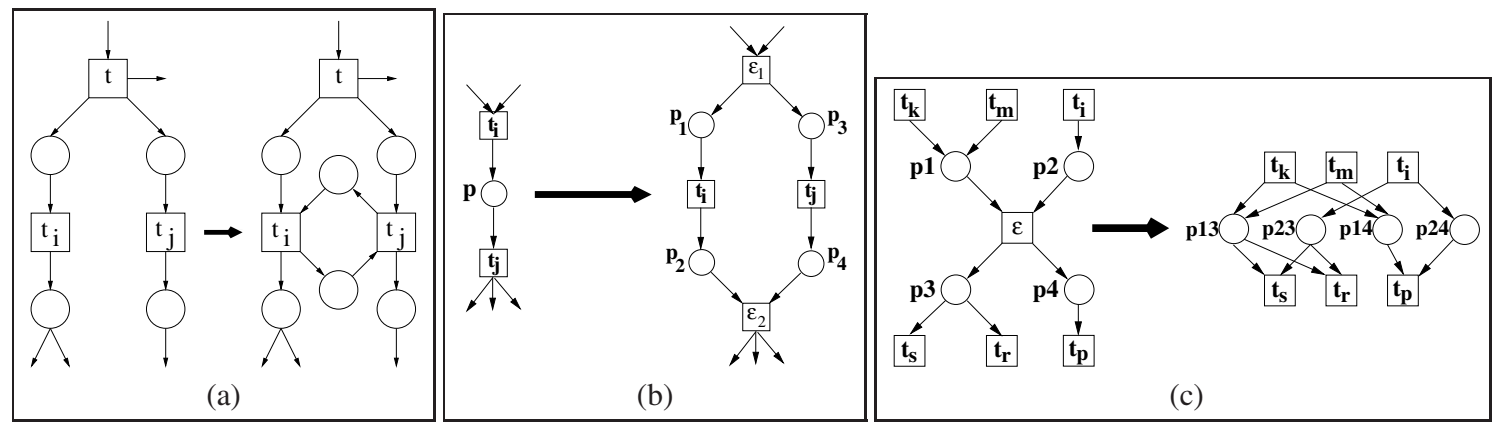

Figure 7. (a) Concurrency reduction, (b) increase of concurrency, (c) transition elimination.

(Da, La and Za) and three output signals (Dr, Lr and Zr). The specification is shown in Figure 8(a). This STG does not have the CSC property. The tool petrify automatically inserts two signals to solve CSC conflicts.

Figure 8(d) shows the STG after being transformed by the structural encoding rules. The new internal signals $s_{0} \ldots s_{14}$ correspond to the 15 places in the initial specification.

From the STG in Figure 8(d), internal signals are greedily removed until CSC conflicts appear. The resulting STG and the corresponding equations (35 literals) are shown in Figure 9(a). Figure 9(b) reports one of the intermediate solutions (31 literals) explored after obtaining the solution in Figure 9(a).

Figures 8(b) and 8(c) depict the final STG, the Boolean equations and the circuit after applying the transformations and doing logic synthesis. This solution, which has been obtained mechanically, is identical to the one generated by petrify.

\subsection{Experimental results}

The synthesis strategy described above has been applied to a set of benchmarks. Initially, none of the specifications had the CSC property. The results are reported in Table 1.

The columns labeled with "petrify" indicate the characteristics of the circuit obtained by the tool petrify. The number of inserted signals to solve CSC conflicts and the number of literals of the Boolean equations are reported.

The columns labeled with "struct. encoding" report the characteristics of the circuit after having applied steps 1 (encoding) and 2 (elimination of internal signals) of the synthesis strategy. It is interesting to observe that the number of signals required to solve CSC conflicts when using the "local" encoding provided by the places is significantly larger than the number of signals required when "global" encoding methods are used.

The results of the final circuit, after having explored the design space with the set of transformations, are reported in the columns labeled "str. enc. + optim.". It can be

\begin{tabular}{c|c||c|c||c|c||c|c}
\cline { 3 - 8 } \multicolumn{2}{c||}{} & \multicolumn{2}{c||}{ petrify } & \multicolumn{2}{c||}{ struct. encoding } & \multicolumn{2}{c}{ str. enc. + optim. } \\
\hline benchmark & states & \#CSC & lit. & \#CSC & lit. & \#CSC & lit. \\
\hline \hline adfast & 44 & 2 & 14 & 5 & 35 & 2 & 14 \\
\hline vme-fc-read & 14 & 1 & 8 & 2 & 14 & 1 & 8 \\
\hline nak-pa & 56 & 1 & 18 & 3 & 35 & 1 & 18 \\
\hline m-read1 & 1882 & 1 & 38 & 2 & 43 & 1 & 40 \\
\hline m-read2 & 8932 & 8 & 68 & 13 & 95 & 10 & 70 \\
\hline duplicator & 20 & 2 & 18 & 5 & 36 & 3 & 18 \\
\hline mmu & 174 & 3 & 29 & 7 & 53 & 3 & 34 \\
\hline seq8 & 36 & 4 & 47 & 22 & 147 & 4 & 47 \\
\hline
\end{tabular}

Table 1. Experimental results.

observed that the quality of the solution can be highly improved by playing with the concurrency of the internal signals. In many cases, the obtained result is the same as the one generated by petrify. In other cases, the results are similar but with more internal signals than the ones inserted by petrify(e.g. master-read2, duplicator). This corroborates a known fact that states that the reduction of internal signals does not always implies an improvement on the quality of the circuit.

The most important fact that can be deduced from this table is that the method proposed in this paper can compete with the existing synthesis tools. Moreover, for the class of FCLSPNs, this method can guarantee and produce an implementation in extremely low CPU times ${ }^{2}$. Until now, no other methods are known that can guarantee an implementation for STGs with underlying FCLSPNs.

\section{Conclusions}

Methods for the synthesis of systems whose complexity does not depend on the size of the state space are crucial to face the design of complex asynchronous circuits.

This paper has presented an approach for the synthesis of asynchronous controllers from STGs. The main features

\footnotetext{
${ }^{2}$ The lack of automation in the application of the transformations did not allow a fair report of CPU times. However, this fact became evident in previous works in this area [24]
} 


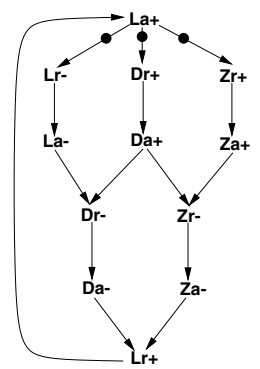

(a)

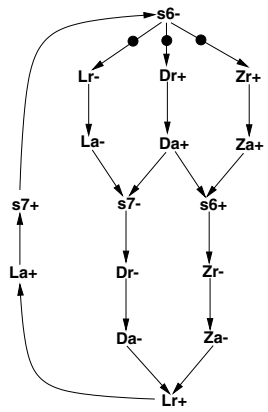

(b)

$$
\begin{aligned}
D r & =s_{7}\left(D a+\overline{s_{6}}\right) \\
L r & =\overline{D a} s_{6} \overline{Z a} \\
Z r & =\overline{s_{6}} \\
s_{6} & =\left(D a+\overline{s_{7}}\right)\left(Z a+s_{6}\right) \\
s_{7} & =L a+\overline{D a} s_{7}
\end{aligned}
$$

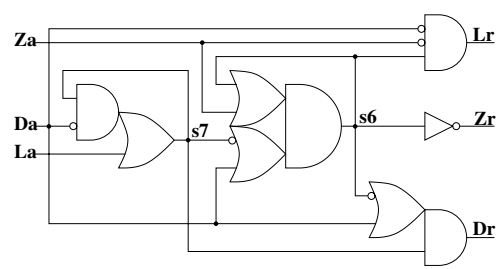

(c)

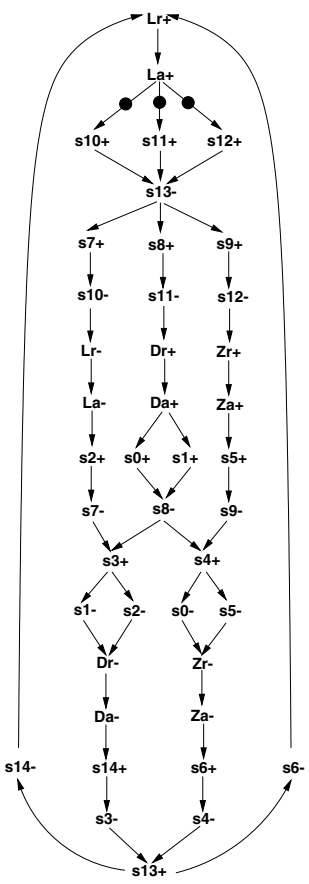

(d)

Figure 8. adfast

of the method are: (1) an implementation is guaranteed and (2) the complexity of the method is polynomial on the size of the specification.

This work is a first step towards a complete automation of the design flow through the exploration of multiple configurations that preserve some equivalence with the original specification. This exploration should allow to find good trade-offs between the size of the implementation and its performance.

\section{References}

[1] K. v. Berkel. Handshake Circuits: an Asynchronous Architecture for VLSI Programming, volume 5 of International Series on Parallel Computation. Cambridge University Press, 1993.

[2] G. Berthelot. Checking Properties of Nets Using Transformations. In G. Rozenberg, editor, Advances in Petri Nets 1985, volume 222 of Lecture Notes in Computer Science, pages 19-40. Springer-Verlag, 1986.

[3] I. Blunno and L. Lavagno. Automated synthesis of micropipelines from behavioral Verilog HDL. In Proc. International Symposium on Advanced Research in Asynchronous Circuits and Systems, pages 84-92. IEEE Computer Society Press, Apr. 2000.

[4] S. M. Burns and A. J. Martin. Syntax-directed translation of concurrent programs into self-timed circuits. In J. Allen and F. Leighton, editors, Advanced Research in VLSI, pages 35-50. MIT Press, 1988.
[5] T.-A. Chu and L. A. Glasser. Synthesis of self-timed control circuits form graphs: An example. In Proc. International Conf. Computer Design (ICCD), pages 565-571. IEEE Computer Society Press, 1986.

[6] J. Cortadella, M. Kishinevsky, A. Kondratyev, L. Lavagno, and A. Yakovlev. Petrify: a tool for manipulating concurrent specifications and synthesis of asynchronous controllers. IEICE Transactions on Information and Systems, E80-D(3):315-325, Mar. 1997.

[7] J. Cortadella, M. Kishinevsky, A. Kondratyev, L. Lavagno, and A. Yakovlev. A region-based theory for state assignment in speed-independent circuits. IEEE Transactions on Computer-Aided Design, 16(8):793-812, Aug. 1997.

[8] R. David. Modular design of asynchronous circuits defined by graphs. IEEE Transactions on Computers, 26(8):727737, Aug. 1977.

[9] A. Davis and S. M. Nowick. An introduction to asynchronous circuit design. In A. Kent and J. G. Williams, editors, The Encyclopedia of Computer Science and Technology, volume 38. Marcel Dekker, New York, Feb. 1998.

[10] J. Desel and J. Esparza. Free Choice Petri Nets. Cambridge University Press, Cambridge, Great Britain, 1995.

[11] J. C. Ebergen. Translating Programs into Delay-Insensitive Circuits. PhD thesis, Dept. of Math. and C.S., Eindhoven Univ. of Technology, 1987.

[12] R. M. Fuhrer. Sequential optimization of asynchronous and synchronous finite-state machines. $\mathrm{PhD}$ thesis, Columbia University, NY, 1999.

[13] S. T. Jung and C. S. Jhon. Direct synthesis of efficient speed-independent circuits from deterministic signal transition graphs. In Proc. International Symposium on Circuits and Systems, pages 307-310, June 1994. 


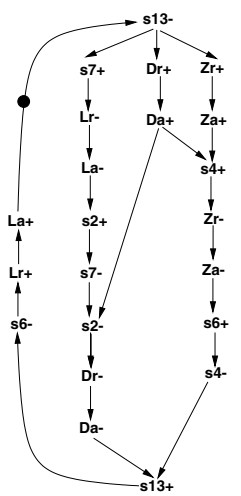

$$
\begin{aligned}
D r & =\overline{s_{13}} L a+s_{2}+s_{7} \\
L r & =\overline{s_{7}} L a+s_{13} \overline{s_{6}} \\
Z r & =\overline{s_{13}} \overline{s_{6}} \overline{s_{4}} \\
s_{2} & =\overline{D a} s_{2}+s_{7} \overline{L a} \\
s_{4} & =Z a\left(\overline{L r} \overline{s_{2}} \overline{s_{7}}+D a\right)+s_{4} \overline{s_{6}} \\
s_{6} & =\overline{s_{13}} s_{6}+\overline{Z a} s_{4} \\
s_{7} & =\overline{s_{13}} L r+\overline{s_{2}} s_{7} \\
s_{13} & =\overline{s_{4}} s_{6} \overline{D a}+s_{13} \overline{L a}
\end{aligned}
$$

(a)

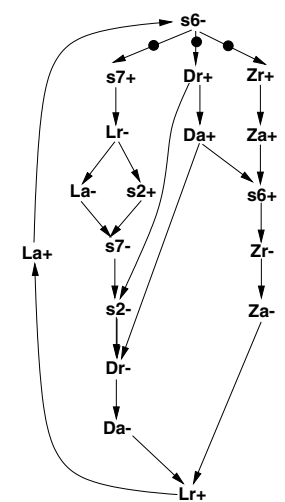

$$
\begin{aligned}
D r & =L a\left(\overline{s_{6}}+D r\right)+\overline{D a} D r+s_{2}+s_{7} \\
L r & =\overline{D a} s_{6} \overline{Z a}+L r \overline{s_{7}} \\
Z r & =\overline{s_{6}} \\
s_{2} & =\overline{D r} s_{2}+\overline{L r} s_{7} \\
s_{6} & =D a\left(s_{6}+Z a\right)+\overline{L a}\left(\overline{D r} Z a \overline{s_{2}} \overline{s_{7}}+s_{6}\right) \\
s_{7} & =L a\left(\overline{s_{6}}+D a\right)+\overline{s_{2}} s_{7}
\end{aligned}
$$

(b)

Figure 9. Intermediate solutions in the design space.

[14] M. Kishinevsky, A. Kondratyev, A. Taubin, and V. Varshavsky. Concurrent Hardware: The Theory and Practice of Self-Timed Design. Series in Parallel Computing. John Wiley \& Sons, 1994.

[15] T. Kolks, S. Vercauteren, and B. Lin. Control resynthesis for control-dominated asynchronous designs. In Proc. International Symposium on Advanced Research in Asynchronous Circuits and Systems, Mar. 1996.

[16] A. Kondratyev, J. Cortadella, M. Kishinevsky, E. Pastor, O. Roig, and A. Yakovlev. Checking Signal Transition Graph implementability by symbolic BDD traversal. In Proc. European Design and Test Conference, pages 325332, Paris, France, Mar. 1995.

[17] S. C. Leung and H. F. Li. A syntax-directed translation for the synthesis of delay-insensitive circuits. IEEE Transactions on VLSI Systems, 2(2):196-210, June 1994.

[18] R. Milner. A calculus of communication systems. In Lecture Notes in Computer Science, volume 92. Springer-Verlag, 1980.

[19] R. K. Morizawa and T. Nanya. A four-phase handshaking asynchronous controller specification style and its idlephase optimization. In Proc. International Conference on Chip Design Automation, 16th World Computer Congress, pages 439-447, Beijing, China, Dec. 2000.

[20] D. E. Muller and W. S. Bartky. A theory of asynchronous circuits. In Proceedings of an International Symposium on the Theory of Switching, pages 204-243. Harvard University Press, Apr. 1959.

[21] T. Murata. Petri Nets: Properties, analysis and applications. Proceedings of the IEEE, pages 541-580, Apr. 1989.

[22] C. J. Myers, T. G. Rokicki, and T. H.-Y. Meng. Automatic synthesis of gate-level timed circuits with choice. In $A d$ - vanced Research in VLSI, pages 42-58. IEEE Computer Society Press, 1995.

[23] E. Pastor and J. Cortadella. An efficient unique state coding algorithm for signal transition graphs. In Proc. International Conf. Computer Design (ICCD), pages 174-177, Oct. 1993.

[24] E. Pastor, J. Cortadella, A. Kondratyev, and O. Roig. Structural methods for the synthesis of speed-independent circuits. IEEE Transactions on Computer-Aided Design, 17(11):1108-1129, Nov. 1998.

[25] M. A. Peña and J. Cortadella. Combining process algebras and Petri nets for the specification and synthesis of asynchronous circuits. In Proc. International Symposium on Advanced Research in Asynchronous Circuits and Systems. IEEE Computer Society Press, Mar. 1996.

[26] L. Y. Rosenblum and A. V. Yakovlev. Signal graphs: from self-timed to timed ones. In Proceedings of International Workshop on Timed Petri Nets, pages 199-207, Torino, Italy, July 1985. IEEE Computer Society Press.

[27] S.-Y. Tan, S. B. Furber, and W.-F. Yen. The design of an asynchronous VHDL synthesizer. In Proc. Design, Automation and Test in Europe (DATE). IEEE Computer Society Press, Feb. 1998.

[28] V. I. Varshavsky, editor. Self-Timed Control of Concurrent Processes: The Design of Aperiodic Logical Circuits in Computers and Discrete Systems. Kluwer Academic Publishers, Dordrecht, The Netherlands, 1990.

[29] C. Ykman-Couvreur, B. Lin, G. Goossens, and H. D. Man. Synthesis and optimization of asynchronous controllers based on extended lock graph theory. In Proc. European Conference on Design Automation (EDAC), pages 512-517. IEEE Computer Society Press, Feb. 1993. 